\title{
Suscetibilidade Diferencial de Plantas Daninhas do Gênero Amaranthus AOS HERBICIDAS TRIFLOXYSULFURON-SODIUM E ChLorimuron-EThYL ${ }^{1}$
}

\author{
Differential Susceptibility of Amaranthus Genus Weed Species to the Herbicides \\ Trifloxysulfuron-Sodium and Chlorimuron-Ethyl
}

\author{
CARVALHO, S.J.P. ${ }^{2}$, BUISSA, J.A.R. ${ }^{3}$, NICOLAI, M. ${ }^{4}$, LÓPEZ-OVEJERO, R.F. ${ }^{5}$ e \\ CHRISTOFFOLETI, P.J. ${ }^{6}$
}

\begin{abstract}
RESUMO - Este trabalho teve por objetivo avaliar a suscetibilidade de cinco espécies de plantas daninhas do gênero Amaranthus a herbicidas aplicados em pós-emergência. As espécies avaliadas foram: A. deflexus (caruru-rasteiro), A. hybridus (caruru-roxo), A. retroflexus (carurugigante), A. spinosus (caruru-de-espinho) e A. viridis (caruru-de-mancha). O trabalho foi dividido em duas fases. Na primeira, as espécies de plantas daninhas foram submetidas à aplicação de 12 tratamentos herbicidas em pós-emergência. Na segunda, os herbicidas trifloxysulfuronsodium e chlorimuron-ethyl foram avaliados com a metodologia de curvas de dose-resposta, repetida duas vezes. Os herbicidas foram aplicados sobre plantas com 5-6 folhas e as doses utilizadas na segunda fase foram: 16D, 4D, D, 1/4D, 1/16D, 1/64D e ausência do produto, em que $\mathrm{D}$ é a dose recomendada de cada herbicida. As doses utilizadas (D) foram de 3,75 e $7,5 \mathrm{~g} \mathrm{ha}^{-1}$ para o herbicida trifloxysulfuron e 12,5 e 17,5 $\mathrm{g} \mathrm{ha}^{-1}$ para chlorimuron, na primeira e na segunda condução, respectivamente. Na primeira fase, foram avaliados o controle percentual e a massa seca das parcelas aos 20 dias após a aplicação (DAA); na segunda, avaliou-se o controle percentual aos 20 DAA. As espécies de Amaranthus avaliadas neste trabalho apresentaram diferenças de suscetibilidade aos herbicidas aplicados em pós-emergência, principalmente ao trifloxysulfuron e ao chlorimuron, em que $A$. deflexus foi a espécie menos suscetivel, seguido por A. spinosus, A. viridis, A. hybridus e A. retroflexus.
\end{abstract}

Palavras-chave: caruru, controle, algodão, soja, dose-resposta.

\begin{abstract}
This work aimed to evaluate the susceptibility of five Amaranthus genus weed species to herbicides applied post-emergence. The species evaluated were: A. deflexus, A. hybridus, A. retroflexus, A. spinosus and A. viridis. The work was divided into two phases. In the first phase, weed species were submitted to post-emergence application of 12 herbicide treatments. In the second phase, the herbicides trifloxysulfuron-sodium and chlorimuron-ethyl were evaluated using the methodology of dose-response curves, repeated twice. The herbicides were applied on plants with 5-6 leaves and the rates used in the second phase were: 16R, 4R, R, 1/4R, 1/16R, 1/64R and product absence, where $R$ is the recommended rate for each herbicide. The rates used $(R)$ were 3.75 and $7.5 \mathrm{~g} \mathrm{ha}^{-1}$ for trifloxysulfuron and 12.5 e $17.5 \mathrm{~g} \mathrm{ha}^{-1}$ for chlorimuron, in the first and second phase, respectively. In the first phase, percent control and dry mass of the pots at 20 days after application (DAA) were evaluated; in the second phase, percent control was evaluated at 20 DAA. The species of Amaranthus that were evaluated presented in this work presented differences of susceptibility to post-emergence applied herbicides, principally to trifloxysulfuron and chlorimuron, with $\mathbf{A}$. deflexus being the least susceptible species, followed by A. spinosus, A. viridis, A. hybridus and A. retroflexus.
\end{abstract}

Keywords: pigweed, control, cotton, soybean, dose-response.

\footnotetext{
Recebido para publicação em 21.3.2006 e na forma revisada em 4.8.2006.

2 Eng.-Agr., Mestrando em Fitotecnia na Escola Superior de Agricultura "Luiz de Queiroz - ESALQ/USP, Caixa Postal 09, 13418-900 Piracicaba-SP, Bolsista FAPESP <sjpcarvalho@yahoo.com.br>; ${ }^{3}$ Eng.-Agr., Doutorando em Fitotecnia na ESALQ/USP, Bolsista CNPq, <marcelon@esalq.usp.br>; ${ }^{4}$ Aluno de Graduação em Engenharia Agronômica da ESALQ/USP, <guto@esalq.usp.br>; ${ }^{5}$ Eng.-Agr., Doutorando em Fitotecnia na ESALQ/USP <rfloveje @ esalq.usp.br>; ${ }^{6}$ Professor Associado do Departamento de Produção Vegetal, ESALQ/USP, <pjchrist@esalq.usp.br>.
}

Planta Daninha, Viçosa-MG, v. 24, n. 3, p. 541-548, 2006 


\section{INTRODUÇÃO}

No mundo, existem cerca de 60 espécies de plantas classificadas no gênero Amaranthus (carurus), e aproximadamente 10 destas têm importância como plantas daninhas das lavouras brasileiras (Kissmann \& Groth, 1999). Os carurus estão presentes em grande parte das áreas agrícolas do país; dentre as espécies mais comuns, podem-se destacar: A. deflexus (caruru-rasteiro), A. hybridus (caruru-roxo), A. lividus (caruru-folha-de-cuia), A. retroflexus (caruru-gigante), A. spinosus (caruru-de-espinho) e A. viridis (caruru-de-mancha).

Quando infestam as lavouras, os carurus competem com as culturas por água, luz e nutrientes; reduzem a quantidade e a qualidade do produto colhido; e, principalmente as espécies de grande porte, interferem nos procedimentos de colheita (Klingaman \& Oliver, 1994; Knezevic et al., 1997; Rowland et al., 1999). Ainda, o manejo das espécies de Amaranthus pode ser dificultado em virtude de apresentarem extenso período de germinação, rápido crescimento, grande produção e viabilidade de sementes (Horak \& Loughin, 2000).

Atualmente, o principal método de controle de plantas daninhas em culturas agrícolas é o químico, por meio da aplicação de herbicidas em pré ou pós-emergência das plantas daninhas e/ou das culturas. Guo \& Al-Khatib (2003) comentam que a aplicação de herbicidas em pós-emergência apresenta vantagens, visto que as plantas já emergiram e podem ser identificadas. Contudo, essa condição de controle está sujeita à influência de fatores relacionados com o tamanho das plantas no instante da aplicação e, principalmente, com a variabilidade na resposta de controle das diferentes espécies, que, por vezes, podem comprometer a eficácia dos produtos (Mayo et al., 1995).

Particularmente para o gênero Amaranthus, em poucos casos as aplicações são feitas sobre plantas identificadas corretamente. Devido à dificuldade de identificação das plantas jovens, em geral, as diferentes espécies dessas plantas daninhas são generalizadas simplesmente como "carurus" (Ahrens et al., 1981; Mayo et al., 1995).

No entanto, freqüentemente, espécies de um mesmo gênero ou família de plantas têm suscetibilidade diferenciada a um mesmo herbicida (Gosset \& Toler, 1999), de forma que as generalizações devem ser evitadas. Assim, este trabalho foi conduzido com o objetivo de avaliar a suscetibilidade de cinco espécies de plantas daninhas do gênero Amaranthus, com ocorrência no Brasil, a alguns dos principais herbicidas com recomendação para aplicações em pós-emergência.

\section{MATERIAL E MÉTODOS}

Os experimentos foram conduzidos em casa de vegetação do Departamento de Produção Vegetal da ESALQ/USP, no município de Piracicaba, São Paulo, entre março e outubro de 2005. As plantas daninhas do gênero Amaranthus utilizadas no trabalho foram: A. deflexus (caruru-rasteiro), A. hybridus (caruru-roxo), A. retroflexus (caruru-gigante), A. spinosus (caruru-de-espinho) e A. viridis (caruru-de-mancha).

Sementes de A. hybridus, A. spinosus e A. viridis foram adquiridas comercialmente. As sementes de $A$. deflexus e $A$. retroflexus foram coletadas em áreas infestadas da ESALQ (A. deflexus) e em áreas agrícolas dos municípios de Miguelópolis e Guará-SP (A. retroflexus). As espécies foram identificadas e as sementes acondicionadas em sacos de papel, em local seco, à temperatura ambiente, até o início da instalação dos experimentos.

O trabalho foi dividido em duas fases. A primeira teve por objetivo realizar uma avaliação geral da suscetibilidade das espécies de Amaranthus a alguns herbicidas com recomendação para aplicação em pós-emergência. O experimento constou de um esquema fatorial entre as cinco espécies de plantas daninhas e 12 tratamentos herbicidas (Tabela 1). Os herbicidas foram escolhidos em função da sua representatividade em aplicações pósemergentes nas culturas de cana-de-açúcar (2,4-D e ametryn), soja (lactofen, fomesafen, imazethapyr e chlorimuron-ethyl), algodão (trifloxysulfuron-sodium e pyrithiobac-sodium) e milho (nicosulfuron, mesotrione e atrazine). Na segunda fase, utilizou-se a metodologia de curvas de dose-resposta para caracterizar a suscetibilidade das espécies aos dois herbicidas que apresentaram as maiores diferenças interespecíficas na primeira fase 
Tabela 1 - Tratamentos herbicidas aplicados sobre cinco espécies de plantas daninhas do gênero Amaranthus. Piracicaba-SP, 2005

\begin{tabular}{|c|c|c|c|c|}
\hline \multicolumn{2}{|c|}{ Tratamento } & \multirow{2}{*}{$\begin{array}{l}\text { Adjuvante } \\
(\%-v / v)\end{array}$} & \multicolumn{2}{|c|}{ Dose } \\
\hline $\begin{array}{l}\text { Produto comercial } \\
\text { (p.c.) }\end{array}$ & $\begin{array}{c}\text { Ingrediente ativo } \\
\text { (i.a.) }\end{array}$ & & mL ou g p.c. ha ${ }^{-1}$ & g i.a. ha ${ }^{-1}$ \\
\hline \multicolumn{2}{|c|}{ Testemunha } & - & - & - \\
\hline DMA 806 & $2,4-\mathrm{D}$ & - & 800 & 536,0 \\
\hline Cobra & lactofen & - & 500 & 120,0 \\
\hline Flex & fomesafen & Agral 1,00 & 600 & 150,0 \\
\hline Pivot & imazethapyr & Agral 0,50 & 700 & 74,2 \\
\hline Classic & chlorimuron-ethyl & Agral 0,50 & 50 & 12,5 \\
\hline Envoke & trifloxysulfuron-sodium & Answer 0,20 & 5 & 3,75 \\
\hline Staple & pyrithiobac-sodium & Answer 0,25 & 200 & 56,0 \\
\hline Sanson & nicosulfuron & - & 600 & 24,0 \\
\hline Callisto & mesotrione & Assist 0,50 & 250 & 100,0 \\
\hline Gesapax & ametryn & Assist 1,00 & 2.000 & $1.000,0$ \\
\hline Gesaprim & atrazine & Assist 1,00 & 2.000 & $1.000,0$ \\
\hline
\end{tabular}

(trifloxysulfuron-sodium e chlorimuron-ethyl). Os critérios de seleção dos dois produtos foram: maiores diferenças absolutas, significância aos testes estatísticos em todas as variáveis, controles inferiores a $80 \%$ e massa seca residual superior a $20 \%$. Nessa fase, as doses utilizadas foram: 16D, 4D, D, 1/4D, 1/16D, $1 / 64 \mathrm{D}$ e ausência dos produtos, em que D é a dose recomendada de cada herbicida. A segunda fase foi repetida duas vezes e as doses utilizadas (D) foram de 3,75 e 7,5 $\mathrm{g} \mathrm{ha}^{-1}$ para o herbicida trifloxysulfuron-sodium e 12,5 e $17,5 \mathrm{~g} \mathrm{ha}^{-1}$ para chlorimuron-ethyl, na primeira e na segunda condução, respectivamente.

O delineamento experimental utilizado foi o de blocos ao acaso, com quatro repetições. As parcelas experimentais constaram de vasos plásticos com capacidade para 0,5 L, preenchidos com substrato comercial acrescido de $2 \mathrm{~g}$ do fertilizante 10:10:10 (N: $\left.\mathrm{P}_{2} \mathrm{O}_{5}: \mathrm{K}_{2} \mathrm{O}\right)$. As sementes das plantas daninhas foram distribuídas em excesso às parcelas, superficialmente, sendo recobertas por fina camada de substrato $(\approx 2 \mathrm{~mm})$. Posteriormente, as parcelas foram desbastadas, obtendo-se densidade final média de 10 plantas por vaso. Quando as plantas apresentavam, em média, 5-6 folhas, realizou-se a aplicação dos herbicidas.

As pulverizações foram realizadas em câmara de aplicação fechada, utilizando-se de ponta do tipo leque (Teejet 80.02E), com jato calibrado na altura de $0,50 \mathrm{~m}$ da superfície do alvo e um volume relativo de calda equivalente a $200 \mathrm{~L} \mathrm{ha}^{-1}$. Quando necessário, utilizou-se adjuvante conforme a recomendação dos fabricantes. Após a aplicação dos herbicidas, os vasos foram colocados em casa de vegetação e irrigados no dia seguinte, para garantir a adequada absorção foliar.

Na primeira fase, foram avaliados o controle percentual e a massa seca das parcelas aos 20 dias após a aplicação (DAA); na segunda fase, avaliou-se o controle percentual aos 20 DAA. As avaliações de controle foram baseadas em valores extremos de 0 (ausência de controle) e $100 \%$ (morte das plantas). A massa seca foi corrigida para valores percentuais, por meio da comparação da massa obtida nos tratamentos herbicidas com a massa da testemunha, considerada $100 \%$.

Os dados foram inicialmente submetidos à aplicação do teste $\mathrm{F}$ na análise de variância. Em seguida, para a primeira fase, aplicou-se o teste de Tukey (5\%) sobre a decomposição fatorial, com o objetivo de comparar as espécies dentro de cada fator herbicida. Na segunda fase, os dados das duas conduções foram analisados conjuntamente, sendo ajustados ao modelo de regressão não-linear do tipo logístico, proposto por Streibig (1988): 


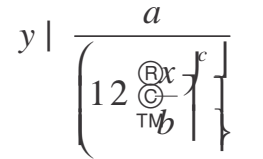

em que: $y$ é o controle percentual; $x$ é a dose do herbicida ( $\mathrm{g} \mathrm{ha}^{-1}$ ); e $a, b$ e $c$ são parâmetros estimados da equação, de tal forma que $a$ é a amplitude existente entre o ponto máximo e o ponto mínimo da variável, $b$ é a dose que proporciona $50 \%$ de resposta da variável e $c$ é a declividade da curva ao redor de $b$.

O modelo logístico apresenta vantagens, uma vez que um dos termos integrantes da equação (b) é uma estimativa do valor de $\mathrm{C}_{50}$ (Christoffoleti, 2002). O $\mathrm{C}_{50}$ (controle de 50\%) é a dose do herbicida, em gramas do ingrediente ativo por hectare, que proporciona o valor de $50 \%$ de controle ou de redução de crescimento da planta daninha (Christoffoleti, 2002; Christoffoleti \& López-Ovejero, 2004). Embora um dos parâmetros do modelo logístico (b) seja uma estimativa do valor de $\mathrm{C}_{50}$, optou-se também por realizar seu cálculo matemático por meio da equação inversa, com base na discussão proposta por Carvalho et al. (2005).

\section{RESULTADOS E DISCUSSÃO}

Na primeira fase, a interação fatorial dos tratamentos herbicidas e das espécies de plantas daninhas foi significativa para as duas variáveis analisadas. Isso indica a existência de pelo menos uma espécie de planta daninha com comportamento diferente do das demais quando submetida a algum herbicida. $\mathrm{Na}$ Tabela 2, observa-se a aplicação do teste de Tukey (5\%) sobre a variável controle percentual aos 20 DAA. Os herbicidas com diferenças interespecíficas significativas foram: imazethapyr, chlorimuron-ethyl, trifloxysulfuron-sodium, pyrithiobac-sodium, nicosulfuron e mesotrione.

Os valores de controle estão em concordância com os dados de massa seca apresentados na Tabela 3, visto que os herbicidas que apresentaram diferenças interespecíficas foram os mesmos para as duas variáveis; as maiores diferenças absolutas estão relacionadas com aplicações de chlorimuron-ethyl e trifloxysulfuron-sodium. Das espécies de plantas daninhas, $A$. deflexus foi a menos suscetivel ao herbicida trifloxysulfuron-sodium, enquanto $A$. deflexus e $A$. spinosus foram as menos suscetiveis ao herbicida chlorimuron-ethyl (Tabelas 2 e 3). Optou-se por não realizar a comparação da eficácia dos herbicidas entre si, uma vez que essa análise não foi o objetivo do trabalho.

Os resultados encontrados estão em concordância com o trabalho conduzido por Gosset \& Toler (1999), que submeteram as espécies A. hybridus e A. palmeri a aplicações de acifluorfen, chlorimuron-ethyl e imazaquin, em pós-emergência. Esses autores verificaram diferenças de controle interespecíficas para herbicidas, em que a espécie $A$. hybridus foi controlada satisfatoriamente por todos os produtos e A. palmeri não o foi.

Mayo et al. (1995) e Sweat et al. (1998) também realizaram experimentos que analisaram a suscetibilidade de espécies de Amaranthus a herbicidas. Em geral, observaram que A. palmeri foi a espécie mais difícil de controlar. Quando as aplicações foram feitas sobre plantas em estádio inicial de desenvolvimento, A. retroflexus foi adequadamente controlada pelos herbicidas lactofen, fomesafen, imazethapyr e chlorimuron-ethyl. Esses dados estão em concordância com aqueles observados nas Tabelas 2 e 3.

Segundo os critérios de seleção dos produtos utilizados na segunda fase do trabalho, os herbicidas escolhidos foram chlorimuron-ethyl e trifloxysulfuron-sodium. Estes herbicidas apresentaram as maiores diferenças interespecíficas, significativas para as duas variáveis; controles percentuais inferiores a $80 \%$ e massas secas residuais superiores a $20 \%$. Para a segunda fase, o controle percentual obtido com a aplicação de múltiplas doses de trifloxysulfuron-sodium e chlorimuron-ethyl sobre as cinco espécies de plantas daninhas está apresentado na Figura 1.

Constatou-se que as espécies de Amaranthus possuem diferenças de suscetibilidade ao herbicida trifloxysulfuron-sodium, em que a espécie mais tolerante foi $A$. deflexus, em concordância com os dados encontrados na primeira fase. Foram encontradas diferenças interespecíficas também para o herbicida chlorimuron-ethyl, principalmente para as subdoses aplicadas, em que, novamente, $A$. deflexus foi a espécie menos suscetivel (Figura 1). 
Tabela 2 - Nível de controle percentual de cinco espécies do gênero Amaranthus submetidas aos tratamentos herbicidas, 20 dias após a aplicação. Piracicaba-SP, 2005

\begin{tabular}{|c|c|c|c|c|c|c|}
\hline \multicolumn{2}{|c|}{ Tratamento } & \multicolumn{5}{|c|}{ 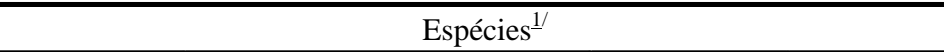 } \\
\hline Herbicida & Dose $\left(\mathrm{g} \mathrm{ha}^{-1}\right)$ & A. deflexus & A. hybridus & A. retroflexus & A. spinosus & A. viridis \\
\hline \multicolumn{2}{|c|}{ Testemunha } & $0,0 \mathrm{a}$ & $0,0 \mathrm{a}$ & $0,0 \mathrm{a}$ & $0,0 \mathrm{a}$ & $0,0 \mathrm{a}$ \\
\hline $2,4-\mathrm{D}$ & 536,00 & $95,8 \mathrm{a}$ & $100,0 \mathrm{a}$ & $100,0 \mathrm{a}$ & $99,4 \mathrm{a}$ & $100,0 \mathrm{a}$ \\
\hline Lactofen & 120,00 & $100,0 \mathrm{a}$ & $100,0 \mathrm{a}$ & $99,0 \mathrm{a}$ & $100,0 \mathrm{a}$ & $99,8 \mathrm{a}$ \\
\hline Fomesafen & 150,00 & 93,8 a & $100,0 \mathrm{a}$ & $98,0 \mathrm{a}$ & 93,0 a & $99,8 \mathrm{a}$ \\
\hline Imazethapyr & 74,20 & $75,0 \mathrm{~b}$ & $99,2 \mathrm{a}$ & $94,8 \mathrm{a}$ & $96,0 \mathrm{a}$ & $99,2 \mathrm{a}$ \\
\hline Chlorimuron-ethyl & 12,50 & $35,0 \mathrm{~b}$ & $97,2 \mathrm{a}$ & $96,6 \mathrm{a}$ & $42,0 \mathrm{~b}$ & $95,0 \mathrm{a}$ \\
\hline Trifloxysulfuron-sodium & 3,75 & $33,0 \mathrm{c}$ & $96,8 \mathrm{a}$ & $93,0 \mathrm{a}$ & $43,0 \mathrm{~b}$ & $97,4 \mathrm{a}$ \\
\hline Pyrithiobac-sodium & 56,00 & $97,2 \mathrm{a}$ & $98,6 \mathrm{a}$ & $93,6 \mathrm{a}$ & $84,0 \mathrm{~b}$ & $100,0 \mathrm{a}$ \\
\hline Nicosulfuron & 24,00 & $90,2 \mathrm{~b}$ & $96,0 \mathrm{ab}$ & $91,4 \mathrm{ab}$ & $96,2 \mathrm{ab}$ & $99,2 \mathrm{a}$ \\
\hline Mesotrione & 100,00 & $81,4 b$ & $99,6 \mathrm{a}$ & $93,6 \mathrm{a}$ & $100,0 \mathrm{a}$ & $97,0 \mathrm{a}$ \\
\hline Ametryn & $1.000,00$ & $100,0 \mathrm{a}$ & $100,0 \mathrm{a}$ & $100,0 \mathrm{a}$ & $100,0 \mathrm{a}$ & $100,0 \mathrm{a}$ \\
\hline \multirow[t]{2}{*}{ Atrazine } & $1.000,00$ & $100,0 \mathrm{a}$ & $100,0 \mathrm{a}$ & $100,0 \mathrm{a}$ & $100,0 \mathrm{a}$ & $100,0 \mathrm{a}$ \\
\hline & \multicolumn{2}{|c|}{$\mathrm{F}_{(\text {herb x esp) }}=32,99 *$} & \multicolumn{2}{|c|}{$\mathrm{DMS}_{\mathrm{esp}}=8,33$} & \multicolumn{2}{|c|}{$\mathrm{CV}(\%)=5,64$} \\
\hline
\end{tabular}

${ }^{1 /}$ Médias seguidas por letras iguais, na linha, não diferem pelo teste de Tukey (5\%).

Tabela 3 - Massa seca percentual de cinco espécies do gênero Amaranthus submetidas aos tratamentos herbicidas, 20 dias após a aplicação. Piracicaba-SP, 2005

\begin{tabular}{|c|c|c|c|c|c|c|}
\hline \multicolumn{2}{|c|}{ Tratamento } & \multicolumn{5}{|c|}{ 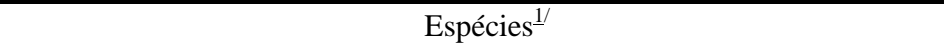 } \\
\hline Herbicida & Dose $\left(\mathrm{g} \mathrm{ha}^{-1}\right)$ & A. deflexus & A. hybridus & A. retroflexus & A. spinosus & A. viridis \\
\hline \multicolumn{2}{|c|}{ Testemunha } & $100,0 \mathrm{a}$ & $100,0 \mathrm{a}$ & $100,0 \mathrm{a}$ & $100,0 \mathrm{a}$ & $100,0 \mathrm{a}$ \\
\hline $2,4-\mathrm{D}$ & 536,00 & $5,7 \mathrm{a}$ & $0,0 \mathrm{a}$ & $0,0 \mathrm{a}$ & $0,4 \mathrm{a}$ & $0,0 \mathrm{a}$ \\
\hline Lactofen & 120,00 & $0,0 \mathrm{a}$ & $0,0 \mathrm{a}$ & $3,2 \mathrm{a}$ & $0,0 \mathrm{a}$ & $0,1 \mathrm{a}$ \\
\hline Fomesafen & 150,00 & $4,0 \mathrm{a}$ & $0,0 \mathrm{a}$ & $5,8 \mathrm{a}$ & $5,7 \mathrm{a}$ & $0,0 \mathrm{a}$ \\
\hline Imazethapyr & 74,20 & $14,9 \mathrm{c}$ & $2,5 \mathrm{ab}$ & $11,4 \mathrm{bc}$ & $3,3 \mathrm{ab}$ & $0,3 \mathrm{a}$ \\
\hline Chlorimuron-ethyl & 12,50 & $40,2 \mathrm{c}$ & $2,1 \mathrm{a}$ & $12,7 \mathrm{~b}$ & $36,8 \mathrm{c}$ & $3,2 \mathrm{ab}$ \\
\hline Trifloxysulfuron-sodium & 3,75 & $42,0 \mathrm{~d}$ & $4,0 \mathrm{ab}$ & $12,8 \mathrm{~b}$ & $25,6 \mathrm{c}$ & $2,1 \mathrm{a}$ \\
\hline Pyrithiobac-sodium & 56,00 & $7,2 \mathrm{ab}$ & $1,7 \mathrm{a}$ & $12,8 \mathrm{~b}$ & $6,4 \mathrm{ab}$ & $0,0 \mathrm{a}$ \\
\hline Nicosulfuron & 24,00 & $10,2 \mathrm{ab}$ & $4,6 \mathrm{a}$ & $14,8 \mathrm{~b}$ & $4,3 \mathrm{a}$ & $0,8 \mathrm{a}$ \\
\hline Mesotrione & 100,00 & $13,2 \mathrm{~b}$ & $0,1 \mathrm{a}$ & $7,5 \mathrm{ab}$ & $0,5 \mathrm{a}$ & $0,7 \mathrm{a}$ \\
\hline Ametryn & $1.000,00$ & $0,0 \mathrm{a}$ & $0,0 \mathrm{a}$ & $0,0 \mathrm{a}$ & $0,0 \mathrm{a}$ & $0,0 \mathrm{a}$ \\
\hline \multirow[t]{2}{*}{ Atrazine } & $1.000,00$ & $0,0 \mathrm{a}$ & $0,0 \mathrm{a}$ & $0,0 \mathrm{a}$ & $0,0 \mathrm{a}$ & $0,0 \mathrm{a}$ \\
\hline & \multicolumn{2}{|c|}{$\mathrm{F}_{(\text {herb x esp) }}=7,22 *$} & \multicolumn{2}{|c|}{$\mathrm{DMS}_{\mathrm{esp}}=9,91$} & \multicolumn{2}{|c|}{$\mathrm{CV}(\%)=41,50$} \\
\hline
\end{tabular}

${ }^{1 /}$ Médias seguidas por letras iguais, na linha, não diferem pelo teste de Tukey (5\%).

Na Tabela 4 encontram-se os parâmetros do modelo logístico ajustados para os controles obtidos com a aplicação das diferentes doses de trifloxysulfuron-sodium e chlorimuron-ethyl sobre as cinco espécies de plantas daninhas. Com esses dados, pôde-se calcular matematicamente os valores de $\mathrm{C}_{50}$ (Carvalho et al., 2005), que caracterizaram os niveis de suscetibilidade das espécies de plantas daninhas aos herbicidas. A ordem decrescente dos valores de $\mathrm{C}_{50}$ para o trifloxysulfuron foi: A. deflexus $(1,141)>A$. spinosus $(0,784)>$ A. viridis $(0,450)>A$. hybridus $(0,297)>$ A. retroflexus $(0,199)$. Para o chlorimuron, a ordem decrescente dos valores de $\mathrm{C}_{50}$ foi: A. deflexus $(5,869)>A$. spinosus $(4,088) \tau$ A. viridis $(3,496)>A$. hybridus $(1,696) \tau$ A. retroflexus $(1,489)$. 

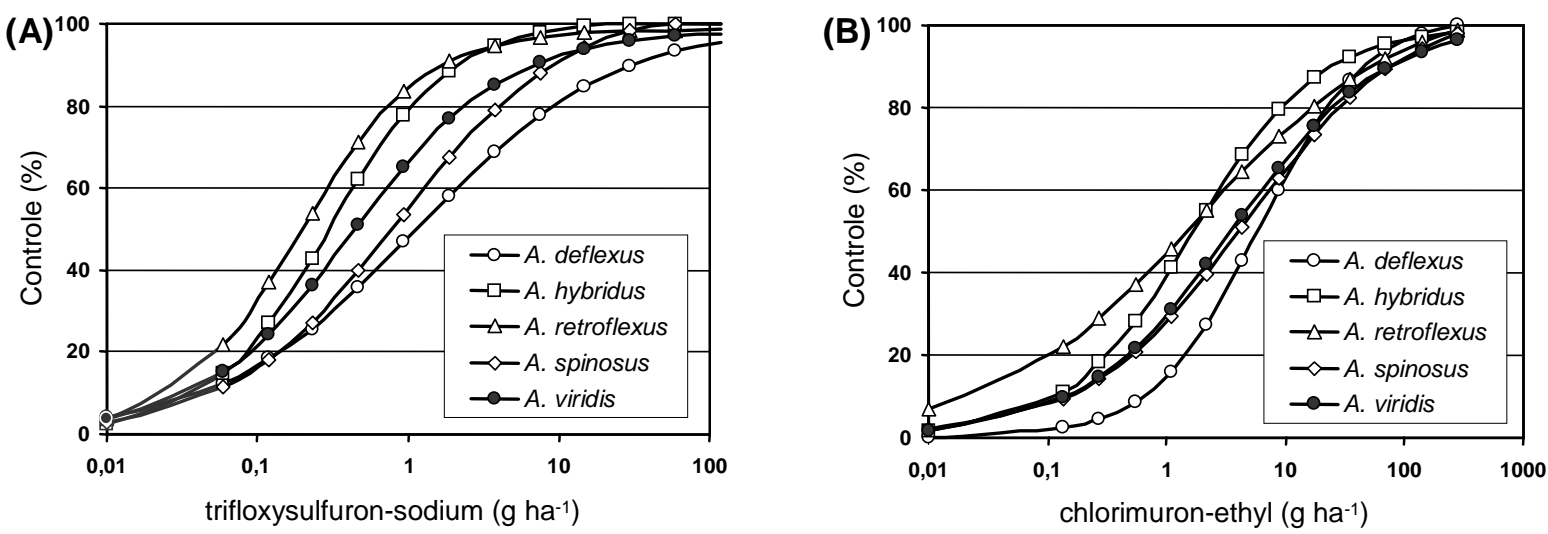

Figura 1 - Nível de controle percentual de cinco espécies do gênero Amaranthus submetidas a diferentes doses dos herbicidas trifloxysulfuron-sodium (A) e chlorimuron-ethyl (B), 20 dias após aplicação. Piracicaba-SP, 2005.

Tabela 4 - Parâmetros do modelo logístico ${ }^{\underline{1}}$, valores da dose que obteve $50 \%$ de controle $\left(\mathrm{C}_{50}\right)$ e coeficientes de determinação $\left(\mathrm{R}^{2}\right)$ para cinco espécies do gênero Amaranthus, submetidas a diferentes doses dos herbicidas trifloxysulfuron-sodium e chlorimuron-ethyl. Piracicaba-SP, 2005

\begin{tabular}{|c|c|c|c|c|c|}
\hline \multirow{2}{*}{ Espécie } & \multicolumn{3}{|c|}{ Parâmetro - Modelo Logístico } & \multirow{2}{*}{$\mathrm{C}_{50}$} & \multirow{2}{*}{$\mathrm{R}^{2}$} \\
\hline & $\mathrm{a}$ & $b$ & $\mathrm{c}$ & & \\
\hline \multicolumn{6}{|c|}{ trifloxysulfuron-sodium } \\
\hline A. deflexus & 100,005 & 1,141 & $-0,663$ & 1,141 & 0,973 \\
\hline A. hybridus & 101,039 & 0,305 & $-0,818$ & 0,297 & 0,955 \\
\hline A. retroflexus & 98,728 & 0,194 & $-1,078$ & 0,199 & 0,955 \\
\hline A. spinosus & 104,505 & 0,876 & $\begin{array}{l}-0,781 \\
-0,01\end{array}$ & 0,784 & 0,980 \\
\hline A. viridis & 98,406 & 0,433 & $-0,864$ & 0,450 & 0,941 \\
\hline \multicolumn{6}{|c|}{ chlorimuron-ethyl } \\
\hline A. deflexus & 102,280 & 6,141 & $-0,986$ & 5,869 & 0,942 \\
\hline A. hybridus & 99,897 & 1,692 & $-0,824$ & 1,696 & 0,974 \\
\hline A. retroflexus & 106,504 & 1,894 & $-0,508$ & 1,489 & 0,952 \\
\hline A. spinosus & 104,925 & 4,724 & $-0,650$ & 4,088 & 0,914 \\
\hline A. viridis & 101,378 & 3,638 & $-0,683$ & 3,496 & 0,938 \\
\hline
\end{tabular}

1/ Modelo: $\mathrm{y}=\left(\mathrm{a} /\left(1+(\mathrm{x} / \mathrm{b})^{\mathrm{c}}\right)\right)$.

A comparação dos valores de $\mathrm{C}_{50}$ comprova a suscetibilidade diferencial das espécies de plantas daninhas do gênero Amaranthus ao trifloxysulfuron-sodium e ao chlorimuronethyl. Resultados de suscetibilidade diferencial de espécies de Amaranthus a herbicidas também foram obtidos por Mayo et al. (1995), Manley et al. (1996), Sweat et al. (1998) e Gosset \& Toler (1999). Contudo, a suscetibilidade diferencial de espécies não se restringe ao gênero Amaranthus. Estudos demonstraram diferenças de suscetibilidade de espécies de Ipomoea ao herbicida bentazon (McClelland et al., 1978; Mathis \& Oliver, 1980) e ao herbicida carfentrazone-ethyl (Christoffoleti et al., 2006); de espécies de Digitaria ao herbicida diuron (Dias et al., 2003); e de espécies de Bidens aos herbicidas chlorimuronethyl e imazethapyr (López-Ovejero et al., 2006).

A diferença de suscetibilidade de espécies de plantas daninhas a herbicidas aplicados em pós-emergência pode estar relacionada com a afinidade enzimática das moléculas; com a absorção, translocação ou exclusão diferencial dos herbicidas; ou mesmo com rotas de detoxificação metabólica. Contudo, maiores estudos 
devem ser conduzidos para esclarecer quais fatores têm participação na resposta diferencial de controle das espécies de plantas daninhas do gênero Amaranthus.

A suscetibilidade diferencial de espécies de plantas daninhas a herbicidas tem implicações diretas sobre o manejo a ser utilizado nas culturas agrícolas. As diferenças interespecíficas de suscetibilidade exigem a correta identificação das espécies que ocorrem nas áreas agrícolas, sobretudo quando em estádio de plântulas, com necessidade da precisa escolha dos herbicidas que serão aplicados. Na cultura do algodão, por exemplo, altas infestações de $A$. deflexus e $A$. spinosus serão mais bem controladas com aplicações de pyrithiobac-sodium. Na cultura da soja, estas mesmas espécies de plantas daninhas serão mais facilmente controladas com aplicações de inibidores da PROTOX (lactofen e fomesafen). Na cultura do milho, atrazine foi o herbicida que apresentou os melhores resultados de controle de qualquer espécie de caruru.

Assim, conclui-se que as espécies de Amaranthus avaliadas possuem diferenças de suscetibilidade aos herbicidas aplicados em pós-emergência, principalmente ao trifloxysulfuron-sodium e ao chlorimuronethyl, em que $A$. deflexus foi a espécie menos suscetivel, seguido por $A$. spinosus, A. viridis, A. hybridus e A. retroflexus.

\section{AGRADECIMENTOS}

Os autores agradecem à Fundação de Amparo à Pesquisa do Estado de São Paulo (FAPESP), pela bolsa de estudos de mestrado concedida ao primeiro autor.

\section{LITERATURA CITADA}

AHRENS, W. H.; WAX, L. M.; STOLLER, E. W. Identification of triazine-resistant Amaranthus spp. Weed Sci., v. 29, n. 3, p. 345-348, 1981.

CARVALHO, S. J. P. et al. Curvas de dose-resposta para avaliação do controle de fluxos de emergência de plantas daninhas pelo herbicida imazapic. Planta Daninha, v. 23, n. 3, p. 535-542, 2005.

CHRISTOFFOLETI, P. J. et al. Carfentrazone-ethyl aplicado em pós-emergência para o controle de Ipomoea spp. e Commelina benghalensis na cultura da cana-de-açúcar. Planta Daninha, v. 24, n. 1, p. 83-90, 2006.
CHRISTOFFOLETI, P. J.; LÓPEZ-OVEJERO, R. F. Definições e situação da resistência de plantas daninhas aos herbicidas no Brasil e no mundo. In: CHRISTOFFOLETI, P. J. (Coord.). Aspectos de resistência de plantas daninhas a herbicidas. 2.ed. Campinas: Associação Brasileira de Ação a Resistência de Plantas aos Herbicidas HRAC-BR, 2004. p. 3-22.

CHRISTOFFOLETI, P. J. Curvas de dose-resposta de biótipos resistente e suscetível de Bidens pilosa L. aos herbicidas inibidores da ALS. Sci. Agric., v. 59, n. 3, p. $513-519,2002$.

DIAS, N. M. P. et al. Absorção e translocação do herbicida diuron por espécies suscetível e tolerante de capim-colchão (Digitaria spp.). Planta Daninha, v. 21, n. 2, p. 293-300, 2003.

GOSSETT, B. J.; TOLER, J. E. Differential control of palmer amaranth (Amaranthus palmeri) and smooth pigweed (Amaranthus hybridus) by postemergence herbicides in soybean (Glycine max). Weed Technol., v. 13, n. 1, p. 165-168, 1999.

GUO, P.; AL-KHATIB, K. Temperature effects on germination and growth of redroot pigweed (Amaranthus retroflexus), Palmer amaranth (A. palmeri), and common waterhemp (A. rudis). Weed Sci., v. 51, n. 6, p. 869-875, 2003.

HORAK, M. J.; LOUGHIN, T. M. Growth analysis of four Amaranthus species. Weed Sci., v. 48, n. 3, p. 347-355, 2000 .

KISSMANN, K. G.; GROTH, D. Plantas infestantes e nocivas. 2.ed. São Paulo: BASF, 1999. v. 2. 978 p.

KLINGAMAN, T. E.; OLIVER, L. R. Palmer amaranth (Amaranthus palmeri) interference in soybeans (Glycine max). Weed Sci., v. 42, n. 4, p. 523-527, 1994.

KNEZEVIC, S. Z.; HORAK, M. J.; VANDERLIP, R. L. Relative time of redroot pigweed (Amaranthus retroflexus) emergence is critical in pigweed-sorghum [Sorghum bicolor (L.) Moench] competition. Weed Sci., v. 45, n. 4, p. 502-508, 1997.

LÓPEZ-OVEJERO, R. F. et al. Resistance and differential susceptibility of Bidens pilosa and B. subalternans biotypes to ALS-inhibiting herbicides. Sci. Agric., v. 63, n. 2, p. 139-145, 2006.

MANLEY, B. S.; WILSON, H. P.; HINES, T. E. Smooth pigweed (Amaranthus hybridus) and livid amaranth (A. lividis) response to several imidazolinone and sulfonylurea herbicides. Weed Technol., v. 10, n. 4, p. 835-841, 1996.

Planta Daninha, Viçosa-MG, v. 24, n. 3, p. 541-548, 2006 
MATHIS, W. D.; OLIVER, L. R. Control of six morningglory (Ipomoea) species in soybeans (Glycine max). Weed Sci., v. 28, n. 4, p. 409-415, 1980.

MAYO, C. M. et al. Differential control of four Amaranthus species by six postmergence herbicides in soybean (Glycine max). Weed Technol., v. 9, n. 1, p. 141-147, 1995.

McCLELLAND, M. R. et al. Responses of six morningglory (Ipomoea) species to bentazon. Weed Sci., v. 26, n. 5, p. 459-464, 1978.
ROWLAND, M. W.; MURRAY, D. S.; VERHALEN, L. M. Full-season Palmer amaranth (Amaranthus palmeri) interference with cotton (Gossipium hirsutum). Weed Sci., v. 47, n. 3, p. 305-309, 1999.

STREIBIG, J. C. Herbicide bioassay. Weed Res., v. 28, n. 6 , p. 479-484, 1988.

SWEAT, J. K. et al. Herbicide efficacy on four Amaranthus species in soybean (Glycine max). Weed Technol., v. 12, n. 2, p. 315-321, 1998. 